Rev. salud pública. 13 (6): 1022-1030, 2011

Modelos/Models

\title{
Incidencia del VIH en la Tuberculosis, en Armenia, Colombia
}

\author{
HIV incidence in cases of tuberculosis in Armenia, Colombia
}

\author{
Milena Corrales-Alvarez ${ }^{1}$, Elmer de la Pava-Salgado ${ }^{2}$ y Luis H. Hurtado-Tobón ${ }^{3}$ \\ 1 Universidad de San Buenaventura. Armenia, Colombia. chamypeco@gmail.com \\ 2 Departamento de Ciencias Ambientales. Universidad Autónoma de Occidente. Cali, Colombia. Cali- \\ Colombia.epava@uao.edu.co \\ 3 Universidad del Quindío. Armenia, Colombia.1hhurtado@uniquindio.edu.co
}

Recibido 24 Febrero 2011/Enviado para Modificación 10 Noviembre 2011/Aceptado 5 Diciembre 2011

\section{RESUMEN}

La Tuberculosis (TBC) y el Síndrome de Inmunodeficiencia Adquirida (SIDA) son patologías que se encuentran muy relacionadas debido a que la TBC es considerada como una de las enfermedades oportunistas del SIDA. Por lo tanto, cuando se investiga el resurgimiento de la TB, es de gran importancia epidemiológica tener en cuenta la influencia que puede tener en su incidencia la prevalencia del SIDA.En este trabajo se desarrolla un procedimiento, con el cual se establece, por medio de un modelo matemático, el efecto del VIH en la incidencia de la TBC en la población de Armenia Q. Los modelos matemáticos se relacionan utilizando un modelo de Función de Transferencia Lineal, lo cual implica desarrollar estimadores insesgados de mínima varianza para los parámetros de cada enfermedad, teniendo en cuenta que se dispone de información secundaria registrada en la Secretaría de Salud del municipio.La aplicación que se hace del modelo para la ciudad de Armenia permite concluir que los enfermos de Tuberculosis en tiempo presente son el resultado de los enfermos de Tuberculosis desde 4 períodos (trimestres) atrás y los enfermos de SIDA desde 2 períodos atrás incluyendo elpresente.El procedimiento que se desarrolla en este trabajo es aplicable a patologías que estén relacionadas.

Palabras Clave: Tuberculosis, VIH, Síndrome de Inmunodeficiencia Adquirida, Incidencia, Modelos Matemáticos, Epidemiología.(fuente: DeCS, BIREME).

\section{ABSTRACT}

Tuberculosis (TB) and the acquired immune deficiency syndrome (AIDS) are closely related diseases because TB is considered one of the opportunistic diseases for AIDS. When investigating the resurgence of TB it is very important epidemiologically to take into account the influence of AIDS prevalence on TB. This paper develops a procedure establishing HIV prevalence in TB for people in Armenia, Quindío, by using a mathematical model. This was done by building two deterministic models 
and relating them through a linear transfer function model. To parameters involved in these models had to be estimated to relate them; a technique was then built from unbiased estimators of minimum variance and secondary information for the parameters of each disease, taking into account the town's Secretariat of Health's secondary information. The model for Armenia led to concluding that tuberculosis resulted from people who had suffered from tuberculosis 4 periods (trimesters) ago and those sick from AIDS 2 periods ago, including the present. The procedure described in this work was seen to be applicable to the pathologies to which it is related.

Key Words: Tuberculosis, HIV, acquired immunodeficiency syndrome, epidemiology, theoretical model (source: MeSH, NLM).

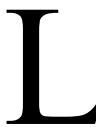

a tuberculosis es una enfermedad infecciosa producida por el bacilo de Koch, llamado también Mycobacterium tuberculosis, este afecta fundamentalmente a los pulmones, pero puede extenderse al sistema nervioso (meningitis), los huesos, los ganglios u otros órganos. Causa muchas muertes y puede dejar secuelas importantes, sobre todo las que ocurren luego de la meningitis tuberculosa (1). Aunque la tuberculosis (TBC) es una enfermedad infecciosa controlable a nivel comunitario y curable de forma individual, dista mucho de ser erradicada. Por otra parte, el Virus de Inmunodeficiencia Humana (VIH) es un virus que ataca el sistema inmunológico, es el causante del Síndrome de Inmunodeficiencia Humana (SIDA), que consiste en la incapacidad del sistema inmunitario para hacer frente a las infecciones y otros procesos patológicos, este virus facilita el desarrollo de nuevas infecciones oportunistas entre las que se encuentra la Tuberculosis (TBC), tumores y otros procesos. "Una persona padece de SIDA cuando su organismo, debido a la inmunodepresión provocada por el VIH, no es capaz de ofrecer una respuesta inmune adecuada contra las infecciones" (2). La TBC es una de las enfermedades asociadas al SIDA más importantes; las alteraciones inmunológicas que acompañan a este síndrome facilitan las formas de TBC de reactivación y la progresión rápida de infección a la enfermedad. En el momento en el que se vislumbraba la posibilidad de erradicar la TBC en algunos países industrializados, la aparición del SIDA amenaza con interferir estas optimistas previsiones que la humanidad tardó en conseguir. Hoy en día se considera que mientras el SIDA no sea controlado, es poco probable que la TBC pueda ser eliminada (3).

En este trabajo se desarrolla una estrategia para evaluar el efecto del VIH en la prevalencia de TBC, utilizando información proporcionada por la Secretaria de Salud del Municipio de Armenia. 
Para evaluar el efecto del VIH en la (incidencia) dela TBC, se construye un modelo determinístico para cada una de las enfermedades y estos dos modelos luego se relacionan mediante un modelo de Función de Transferencia Lineal, que se construye con series de tiempo generadas por los modelos determinísticos. Las series de tiempo consideradas son las correspondientes a Infecciosos para la TBC y Total de Infectados para el VIH, ambas son modeladas por el procedimiento ARIMA.

Un paso previo a la generación de las dos series es la construcción de estimadores insesgados y de mínima varianza para los parámetros de los sistemas de ecuaciones con que se modelan cada una de las enfermedades, teniendo en cuenta que se trata de utilizar información secundaria que se registra en la Secretaría de Salud.

\section{MÉTODOS}

Para determinar la Incidencia del VIH en la TBC en la ciudad de Armenia se construyeron dos modelos matemáticos independientes, teniendo en cuenta el comportamiento de cada patología.

A continuación se muestran los diagramas compartimentales, que permiten comprender la dinámica del VIH [1] y la TBC [2]:

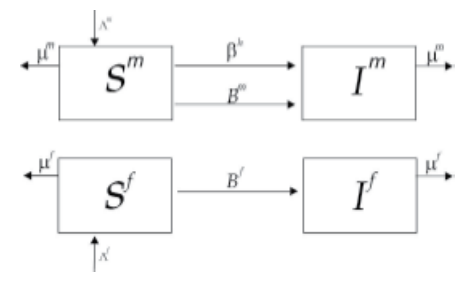

[1]

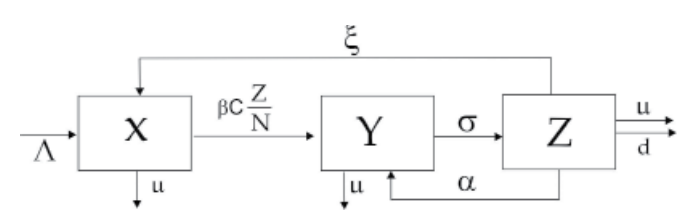

[2]

Para relacionar estos modelos se construyó un Modelo de Función de Transferencia Lineal, para lo que se hizo necesario estimar parámetros con datos, para esto se utilizaron los datos proporcionados por la Secretaría de Salud, datos que son obtenidos de pacientes que presentan dichas patologías y por el Departamento Administrativo Nacional de Estadística (DANE). 
Datos proporcionados para la TBC: Cp: Cultivo positivo, Bp: Baciloscopía positiva,Tr: Transferidos, Sc: Sintomáticos respiratorios, Inc:Investigados e Isc: Inscritos a contactos, Se: Sintomáticosexaminados, $\mathrm{Cr}$ : Casos registrados, $\mathrm{Cu}$ : Curados, Fa: Fallecidos acausa de la enfermedad, Tt: Tratamiento terminado, Fr: Fracasos yAb: Abandonos.

Para el SIDA los datos se indican a continuación. Hhomo: Hombres homosexuales, Hhete: heterosexuales y Hbis: bisexuales infectados, Minf: Mujeres infectadas; además fueron proporcionados datos que corresponden a Na: nacimientos, Fall: fallecimientos y Tp: Total de la población discriminada por sexo (NaM, NaH, FallM, FallH, TpH y TpM).

Ahora se definen las tasas (4) para estimar los parámetros de los modelos (1) y (2), en la siguiente forma:

\begin{tabular}{|l|c|}
\hline \multicolumn{2}{|c|}{ TBC } \\
\hline $\begin{array}{l}\text { Tasa de muerte } \\
\text { a causa de la } \\
\text { enfermedad }\end{array}$ & $\frac{F a}{T p}$ \\
\hline $\begin{array}{l}\text { Tasa de } \\
\text { personas que } \\
\text { no terminan el } \\
\text { tratamiento }\end{array}$ & $\frac{A b}{C r}$ \\
\hline $\begin{array}{l}\text { Tasa de } \\
\text { transferencia del } \\
\text { estado Infectado } \\
\text { al Infeccioso }\end{array}$ & $\frac{B p+T r+I n c+I s c+C p}{S e}$ \\
\hline $\begin{array}{l}\text { Tasa de } \\
\text { contacto }\end{array}$ & $\frac{S c+I n c}{I s c}$ \\
\hline $\begin{array}{l}\text { Tasa de } \\
\text { transmisión }\end{array}$ & $\frac{45}{1000}$ \\
\hline $\begin{array}{l}\text { Tasa de } \\
\text { reclutamiento }\end{array}$ & $\frac{N a}{T p}$ \\
\hline $\begin{array}{l}\text { Tasa de muerte } \\
\text { natural }\end{array}$ & $\frac{F a l l}{T p}$ \\
\hline $\begin{array}{l}\text { Tasa de } \\
\text { curados }\end{array}$ & $\frac{C u}{C r}$ \\
\hline
\end{tabular}

\begin{tabular}{|l|c|}
\hline \multicolumn{2}{|c|}{ VIH } \\
\hline $\begin{array}{l}\text { Tasa de } \\
\text { transmisión } \\
\text { hombres } \\
\text { heterosexuales }\end{array}$ & $\frac{\text { Hhete }+ \text { Hbis }}{T p H}$ \\
\hline $\begin{array}{l}\text { Tasa de } \\
\text { transmisión } \\
\text { hombres } \\
\text { homosexuales }\end{array}$ & $\frac{\text { homo }+ \text { Hbis }}{T p H}$ \\
\hline $\begin{array}{l}\text { Tasa de } \\
\text { transmisión } \\
\text { mujeres }\end{array}$ & $\frac{M \text { inf }}{T p M}$ \\
\hline $\begin{array}{l}\text { Tasa de muertes } \\
\text { mujeres }\end{array}$ & $\frac{\text { FallM }}{T p}$ \\
\hline $\begin{array}{l}\text { Tasa de muerte } \\
\text { hombres }\end{array}$ & $\frac{\text { FallH }}{T p}$ \\
\hline $\begin{array}{l}\text { Tasa nacimiento } \\
\text { de mujeres }\end{array}$ & $\frac{N a M}{T p}$ \\
\hline $\begin{array}{l}\text { Tasa de } \\
\text { nacimiento } \\
\text { hombres }\end{array}$ & $\frac{N a H}{T p}$ \\
\hline $\begin{array}{l}\text { Tasa de formación } \\
\text { de parejas }\end{array}$ & $\frac{T o t a l \text { hogares }}{T p}$ \\
\hline
\end{tabular}


En este modelo se deben estimar las funciones de Formación de Parejas, lo que se realiza con una de las funciones utilizadas en epidemiología (5),

$$
r^{(m, f)}(m, f)=c^{(m, f)} \min (m, f)
$$

donde $c^{(m, f)}$ es una constante positiva, que denota la tasa de formación de parejas para hombres y mujeres respectivamente, estas constantes se estimaron considerando los hogares de la ciudad de Armenia (DANE) y el total de la población.

El estimador para los parámetros está dado por $\hat{\lambda}=-\ln \left(1-\frac{\sum_{i=1}^{r} x_{i}}{\sum_{i=1}^{r} y_{i}}\right)$ donde, $\sum_{i=1}^{r} x_{i}$ es el número de casos positivos en una muestra $\mathrm{y}$

$\sum_{i=1}^{r} y_{i}$ los casos analizados.

Reemplazando datos en el estimador se tienen las estimaciones para los parámetros en los modelos:

\begin{tabular}{|c|c|}
\hline \multicolumn{2}{|c|}{ TBC } \\
\hline$d$ & 0.1107 \\
\hline$\alpha$ & 0.1014 \\
\hline$\sigma$ & 0.4572 \\
\hline$c$ & 0.1534 \\
\hline$\beta$ & 0.4604 \\
\hline$\lambda$ & 0.0199 \\
\hline$\mu$ & 0.006 \\
\hline$\xi$ & 0.066 \\
\hline
\end{tabular}

\begin{tabular}{|l|l|}
\hline \multicolumn{2}{|c|}{$\mathrm{VIH}$} \\
\hline$\lambda^{f}$ & 0.0185 \\
\hline$\lambda^{m}$ & 0.0211 \\
\hline$\mu^{f}$ & 0.0048 \\
\hline$\mu^{m}$ & 0.0073 \\
\hline$\beta^{m}$ & 0.0000346 \\
\hline$\beta^{h}$ & 0.0000259 \\
\hline$\beta^{f}$ & 0.0000221 \\
\hline$r^{f}$ & $7156276779+$ \\
$r^{m}$ & \\
\hline
\end{tabular}


Estimación del Modelo de Función de Transferencia Lineal Mftl

Para la construcción del MFTL se tienen dos series: una que se considera dependiente y otra independiente, como se necesita establecer el efecto que ejerce el VIH en la TBC, entonces la serie independiente es la generada por el VIH y la dependiente es la generada por la TBC.

Así que el modelo de Transferencia Ruido Blanco está dado por

$$
Y_{t}=\frac{0.1075}{1-0.477 B-0.233 B^{2}} X_{t}+\frac{1}{1-1.90601 B-0.953699 B^{2}} a_{t}
$$

o en forma equivalente $=$

$$
\begin{aligned}
& 0.2222 Y_{t-4}+0.0108 Y_{t-3}-1.62980 Y_{t-2}+0.477 Y_{t-1}+0.1025 X_{t-2}-0.2048 X_{t-1}+0.1075 X_{t} \\
& \left.-0.477 B-0.233 B^{2}\right) a_{t}
\end{aligned}
$$

Este modelo muestra que la TBC en un tiempo $t$ depende de ella misma desde cuatro períodos atrás y el VIH influye en el presente y dos períodos atrás.

\section{RESULTADOS}

Una evaluación del modelo de función de transferencia se presenta en la siguiente gráfica, donde se enfrentan los datos proporcionados por la Secretaría de Salud y la predicción del Modelo de Función de Transferencia Lineal.

Figura 1. Evaluación del modelo de Función de Transferencia Lineal

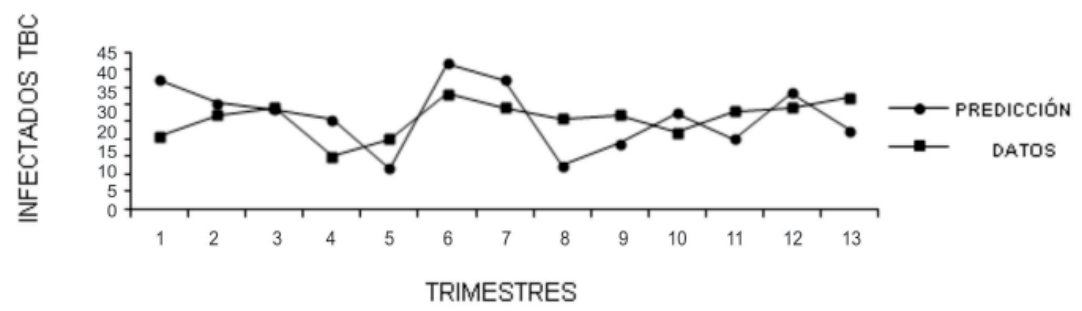


El siguiente esquema gráfico permite visualizar el resultado obtenido por el modelo:

Figura 2. Representación gráfica del efecto del VIH en la TBC

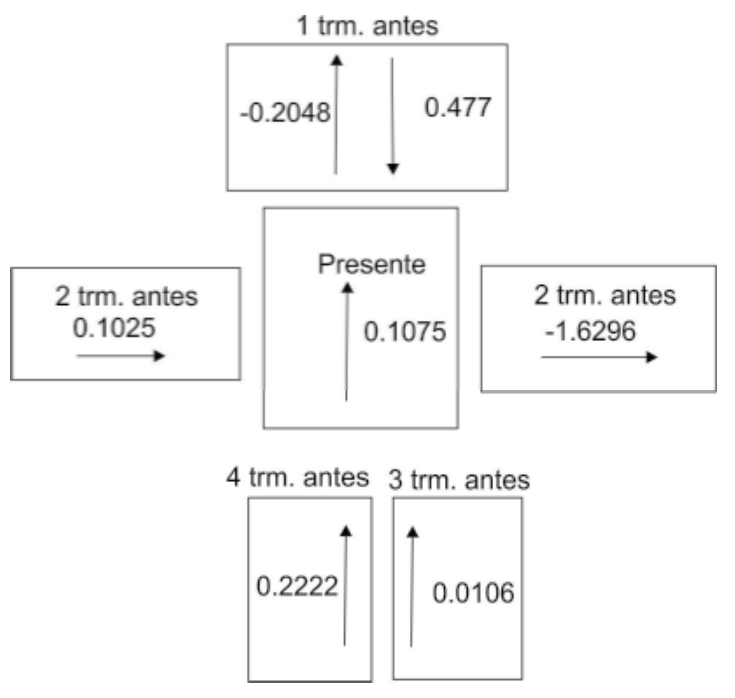

En conclusión una persona enferma en el presente de Tuberculosis, es el resultado de los enfermos de TBC que aparecieron desde cuatro trimestres atrás y las personas infectadas con TBC y VIH desde dos trimestres atrás incluyendo el presente.

\section{DISCUSIÓN}

Con la aparición del VIH en los años 80 se han incrementado los casos de TBC a nivel mundial, es por esto que cuando se habla deTuberculosis es inevitable pensar en SIDA. Debido a la relación existente entre estas dos enfermedades, han surgido diferentes modelos matemáticos que describen el comportamiento conjunto; estos modelos son muy complejos debido a que solo en uno se incluye las dos enfermedades, lo que hace que los cálculos y el número de parámetros sea extenso y el análisis resulte complicado.

Con este trabajo se plantea un procedimiento alternativo más simple para determinar la incidencia del VIH en la TBC en la ciudad de Armenia Quindío, relacionando modelos determinísticos con un modelo estocástico. 
Si se plantea un modelo determinístico para cada enfermedad y se tiene acceso a información secundaria, es posible construir un modelo de Función de Transferencia Lineal, que permite relacionar las dos enfermedades modeladas.

En este trabajo se propone un método para estimar parámetros, que se construye bajo el supuesto «la transición de un estado a otro tiene una distribución de Poisson», esto permite deducir la fuerza de ocurrencia del evento y establecer el estimador insesgado y de mínima varianza, lo que permite aplicar en la realidad modelos determinísticos a partir de información secundaria.

Este trabajo deja abierta las posibilidades para relacionar diferentes enfermedades utilizando esta metodología; un ejemplo podría ser la relación entre desnutrición y enfermedades respiratorias en los niños; además, la técnica para estimar parámetros facilita a la aplicación de los modelos en la realidad

\section{REFERENCIAS}

1. Rossman MD, Mac Gregor RR. Tuberculosis. México D.F.: McGraw Hill Inter. Americana; 1996.

2. Colaboradores de Wikipedia, [Internet]. Disponible en: http://es.wikipedia.org/wiki/Sida. Consultado: Septiembre de 2007.

3. Farreras P, Rozman C, Medicina Interna. $13^{\text {ra }}$ Edición. Barcelona: Mosby/Doymalibros; 1995.

4. Lubkin S, Hsu S, Castillo Chavez C, A Framework for Modeling of Social Traits, Technical Report of BSBCB, Cornell University, U.S. Army Research Office through the Mathematical Sciences Institute of Cornell University; 1991. DAAL03-91C-0027.

5. Castillo ChC, Baojun S. An Overview of Dynamical Models of Tuberculosis. TechnicalReport of BSBCB, Cornell University, Ithaca; 2002. BU-1607-M.

6. Hurtado LH, Salcedo GE, Serrano HJ. Modelos Estadísticos para Información Correlacionada. Cuadernos Biomatemáticas 2. Universidad del Quindío, Facultad de Formación Avanzada e Investigaciones, Programa de Matemáticas y Computación. 1996.

7. De la Pava E. Modelación matemática con estructura de edad del riesgo de infección tuberculosa en la ciudad de Cali. Matemáticas Enseñanza Universitaria, Universidad del Valle. 2008; XVI (.2): 37-56.

8. Castillo Chavez C., Wenzhang H., Li J., On Existence of stable Pairing Distributions, Journal of Mathematical Biology, Springer Verlag, 1996; Vol. 34, p.413-441.

9. Castillo Chavez C., Wenzhang H. y Jia Li; Competitive exclusion in gonorrhea models and other sexually transmitted diseases, Society for Industrial and Applied Mathematics, 1996; Vol. 56 No. 20, p.494-508.

10. Styblo K., Epidemiology of tuberculosis. The Hague: Royal Netherlands Tuberculosis Association, 1991.

11. Castillo Chavez C., Song B., Aparicio J., Tuberculosis models with fast and slow dynamics: the role of close and casual contacts, Mathematical Biosciences, Elsevier, 2002; Vol. 180 , p.187-205. 
12. Porco TC, Small PM, Blower SM, Amplification Dynamics: Predicting the effect of HIV on Tuberculosis Outbreaks, JAIDS Journal of Acquired Immune Deficiency Syndromes, 2001; 28:437-444.

13. Waaler H., Gesser A., Andersen S., The use of mathematical in the study of the epidemiology of tuberculosis, Ame J. Pub HIth, 1962; Vol. 52 (6):1002-1013.

14. May RM, Anderson RM. Transmission dynamics of HIV infection. Nature. 1987; 326: 137142.

15. Keyfitz N. Thematics of sex and marriage. Proceedings of the Sixth Berkeley Symposium on Mathematical Stattistics and Probability. 1949; 4:89-108.

16. Massad E, Burattini NM, Bezerra C, Yang H, Martorano R. Modelling the interaction between AIDS and tuberculosis. Math. Comput. Modelling. 1993; 17 (9): 7-21.

17. Kermack WO, Mckendrick AG. A contribution to the mathematical theory of epidemics. Prod. Roy. Soc A., 1927; 115: 700-721.

18. López MH, Mantilla IP. Un modelo matemático para el estudio de la incidencia del SIDA en la Tuberculosis. Boletín de matemáticas, Nueva Serie. 1998; 2: 81-96.

19. Edelstein KL. Mathematical Models in Biology, First Edition. New York: The Random House Birk hauser Mathematics Series;1988.

20. Box GEP, Jenkins GM. Time Series Analysis. San Francisco. Forecasting and Control. Holden Day; 1976.

21. Christopher M, Martcheva M. Vaccination strategies and backward bifurcation in an agesince-infection structured model. Mathematical Biosciencies. 2002; 177:317-332.

22. Instituto Seccional de Salud del Quindío. Guía de Atención de la Tuberculosis Pulmonar y Extrapulmonar. Technical Report of BSBCB. Cornell University, Ministerio de Salud, Dirección General de Salud Pública; 2005.

23. Modeling Contact Structures in Biology [Internet]. Disponible en http://www.etsu.edu/cas/ math/documents/mixing3.pdf Consultado Abril de 2007.

24. Virus de la Inmunodeficiencia Humana [Internet]. Disponible en: http://www.laescolar.com/ frame_builder.html Consultado Agosto del 2007.

25. Hurtado LH, Salcedo GE. Series Temporales con Aplicaciones a la Epidemiología y a la Ecología. Armenia: Universidad del Quindío; 1996.

26. Huang W, Cook KL, Castillo Chavez C. Stability and bifurcation for a multiple-group model for the dynamics of HIVIAIDS transmission. SIAM J. Appl. Math. 1992; 52(3):835-854.

27. Mood AM, Graybill FA, Boes DC. Introduction to the Theory Of Statistics. Third Edition. McGraw-Hill International Editions; 1974.

28. Micobacterium Tuberculosis [Internet]. Disponible en http://moneras.blogspot.com/2007/05/ un-mundo-microscopico.html Consultado Agosto 2007.

29. Dieckmann O, Heesterbeek $\mathrm{H}$. On the Definition and the computation of the basic ratioin models for infectious diseases in heterogeneous populations. J.Math.Biol. 1990;28(4):365-82.

30. ACI prensa [Internet]. Disponible en:http://www.aciprensa.com/sida. Consultado Septiembre de 2007.

31. Dieckmann O, Heesterbeek H. Mathematical epidemiology of infectious diseases. Chichester: John Wiley and Son Lt; ,2000. 